\title{
Grating-assisted superresolution of slow waves in Fourier space
}

Thomas, N. Le; Houdré, R.; Frandsen, Lars Hagedorn; Fage-Pedersen, Jacob; Lavrinenko, Andrei; Borel, Peter Ingo

Published in:

Physical Review B Condensed Matter

Link to article, DOI:

10.1103/PhysRevB.76.035103

Publication date:

2007

Document Version

Publisher's PDF, also known as Version of record

Link back to DTU Orbit

Citation (APA):

Thomas, N. L., Houdré, R., Frandsen, L. H., Fage-Pedersen, J., Lavrinenko, A., \& Borel, P. I. (2007). Gratingassisted superresolution of slow waves in Fourier space. Physical Review B Condensed Matter, 76(3), 035103. https://doi.org/10.1103/PhysRevB.76.035103

\section{General rights}

Copyright and moral rights for the publications made accessible in the public portal are retained by the authors and/or other copyright owners and it is a condition of accessing publications that users recognise and abide by the legal requirements associated with these rights.

- Users may download and print one copy of any publication from the public portal for the purpose of private study or research.

- You may not further distribute the material or use it for any profit-making activity or commercial gain

- You may freely distribute the URL identifying the publication in the public portal

If you believe that this document breaches copyright please contact us providing details, and we will remove access to the work immediately and investigate your claim 


\title{
Grating-assisted superresolution of slow waves in Fourier space
}

\author{
N. Le Thomas and R. Houdré \\ Institut de Photonique et d'Electronique Quantique, Ecole Polytechnique Fédérale de Lausanne (EPFL), Station 3, \\ CH-1015 Lausanne, Switzerland
}

\author{
L. H. Frandsen, J. Fage-Pedersen, A. V. Lavrinenko, and P. I. Borel \\ COM·DTU-Department of Communications, Optics and Materials, Nano.DTU, Technical University of Denmark, \\ DK-2800 Kongens Lyngby, Denmark
}

(Received 31 May 2007; revised manuscript received 6 June 2007; published 5 July 2007)

\begin{abstract}
We present a far-field optical technique allowing measurements of the dispersion relation of electromagnetic fields propagating under the light cone in photonic nanostructures. It relies on the use of a one-dimensional grating to probe the evanescent tail of the guided field in combination with a high-numerical-aperture Fourierspace imaging setup. A high-resolution spectroscopy of the far-field emission diagram allows us to accurately and efficiently determine the dispersion curve and the group-index dispersion of planar photonic crystal waveguides operating in the slow-light regime.
\end{abstract}

DOI: 10.1103/PhysRevB.76.035103 PACS number(s): 42.70.Qs, 42.25.Bs, 42.30.Kq, 78.67. -n

\section{INTRODUCTION}

The optical information about an object resides in fields radiating in the far-field and in evanescent fields, exponentially vanishing away from the object. For some objects, all the information is entirely contained in the evanescent fields. This situation is found in many classes of structures of fundamental and practical interest, for instance, in nanophotonic structures, such as dielectric nanophotonic wires, ${ }^{1}$ channel plasmon waveguides, ${ }^{2,3}$ and photonic crystal $(\mathrm{PhC})$ structures. ${ }^{4-6}$ In this paper, we demonstrate a general way to convert the information carried by evanescent fields into radiative field suitable for far-field imaging. In a $\mathrm{PhC}$, periodic nanostructurations of the refractive index in a dielectric medium give access to a large variety of original dispersive properties. Such properties are characterized by the dispersion relation $\omega(k)$, in the same way as the energy band diagram contains the essential information about the propagation of the electronic waves in solid-state physics. Molding the dispersion relations is currently the object of intense investigations. One of the main driving forces is the achievement of nanophotonic structures with very slow-light propagation. $^{7-11}$

The characterization of nonradiative electromagnetic excitations (i.e., waves whose wave vector $k$ is larger than the vacuum field wave vector $k_{v}$ ) is difficult and requires complex noninvasive techniques that probe the light with minimal perturbation. Two main interferometric methods have been used up to now. The first relies on the use of specially designed structures and/or Fabry-Pérot interferences observed in the transmission spectrum that allows the determination of the group-index $n_{g}$ and thus, by integration, the dispersion relations. ${ }^{5,6}$ Such technique suffers, however, from uncertainty on the absolute $k$ value, as pointed out by Notomi et $a .^{5}$ In addition, the common use of access waveguides may induce coupled-cavity effects that dramatically complicate the analysis of the Fabry-Pérot interference pattern, particularly in the slow-light regime where impedance mismatch with the access waveguides drastically in- creases. The second approach, which relies more on local experimental techniques, is the heterodyne scanning nearfield optical microscopy (SNOM). ${ }^{12,13}$ It is currently one of the most commonly used methods because of its capability to track the light pulses propagating in the structure and to image objects of subwavelength size. However, the inherent problems related to fiber-tip scanning over large areas, to the heterodyne technique, and to the cumbersome numerical postprocessing invite to explore an alternative physical process able to probe the evanescent fields.

A far-field optical technique using attenuated total reflectance (ATR) has recently been presented by Galli et al. to investigate the dispersion of $\mathrm{PhC}$ waveguides outside of the light cone. ${ }^{14}$ It relies on the use of a high index hemisphere probing the evanescent tail of the propagating mode and converting wave vectors below the light line into propagating ones. By principle, the attenuated reflection method cannot be directly used for the characterization of a structure operating under real condition, i.e., with the excitation beam guided in the planar waveguide. Hemispheric lenses, suffering from intrinsic geometrical aberrations, are also unable to achieve high resolution in the wave vector space, i.e., in the Fourier space. As pointed by the authors themselves, the alignments are extremely critical, the retrieved information very sensitive to the coupling, and no benefit is really gained from SNOM techniques from this point of view. As a result, integrated converters of evanescent to radiative fields are more appealing. Recently, gratings have theoretically been proposed to image subwavelength objects beyond the Rayleigh criterion by using far-field optical diffraction tomography. ${ }^{15}$

We will show that the intrinsic physical properties of slow-light modes propagating below the light cone in planar $\mathrm{PhC}$ waveguides can be measured precisely with conventional Fourier optics. The main concept is to directly integrate the evanescent wave probe, a grating, with the investigated object, which permits to achieve a superresolution of the slow wave in the Fourier space in real operating condition and without cumbersome adjustments. As a result, the experimental dispersion curves for standard as well as for 
specially tailored single-line-defect (W1) $\mathrm{PhC}$ waveguides are accurately determined, as confirmed by good agreement with three-dimensional (3D) plane wave expansion modeling. A very narrow bandwidth excitation source and direct $k$-space spectroscopy provide a fine analysis of the different Bloch modes excited in the photonic structure. The $k$-space spectral characteristics of forward and backward guided modes, as well as propagating Bloch modes of the PhC continuum, are clearly separated. The accurate measurement of the dispersion curve provides a precise determination of the group-index dispersion.

\section{THEORY}

The most noninvasive investigation of the dispersion relations of fields excited in photonic structures and propagating below the light cone is the detection of residual optical losses resulting from disorder in the medium. Static defects in the structure, considered as a small perturbation of the dielectric function of the medium, induce optical losses by scattering of the propagating field, e.g., a plane wave with wave vector $\vec{k}_{i}$. The angular spectrum representation of the scattered field $S\left(\vec{k}, \vec{k}_{i}, \omega\right)$ in $k$ space is generally related to the incident wave vector $\vec{k}_{i}$ via the dispersion relation

$$
S\left(\vec{k}, \vec{k}_{i}, \omega\right)=\sum_{\vec{r}_{n}, \vec{r}_{m}} f\left(\vec{r}_{n}, \vec{r}_{m}\right) \exp \left[j\left(\vec{k}_{i} \cdot \vec{r}_{n}-\vec{k} \cdot \vec{r}_{m}\right)\right]
$$

with $f\left(\vec{r}_{n}, \vec{r}_{m}\right)$ being the complex amplitude corresponding to the propagation between scattering events located at $\vec{r}_{n}$ and $\vec{r}_{m}$. In the first Born approximation, this expression reduces to $S\left(\vec{k}, \vec{k}_{i}, \omega\right)=\widetilde{F}\left[\vec{k}-\vec{k}_{i}(\omega)\right]$ with $\tilde{F}$ being the Fourier transform of the scattering potential. The ability to measure the $\vec{k}-\vec{k}_{i}(\omega)$ dependence versus the angular frequency $\omega$ is the key element in recovering information about the $\vec{k}_{i}(\omega)$ relation in the light cone. The measurement of $S\left(\vec{k}, \vec{k}_{i}, \omega\right)$ for each angular frequency $\omega$ gives, in principle, access to the dispersion curve $\omega\left(k_{i}\right)$ of the incident field, if $f\left(\vec{r}_{n}, \vec{r}_{m}\right)$ or the scattering potential are known, which is not the case when disorder is involved. In contrast to a random distribution of scattering defects, the scattering potential $\widetilde{F}$ is perfectly known for a grating. As a result, $\omega\left(k_{i}\right)$ can be directly deduced from the measurement of $S\left(\vec{k}, \vec{k}_{i}, \omega\right)$, and the dispersion relation [Eq. (1)] translates in a straightforward wave vector conservation rule between the incident wave vector and the wave vector distribution of the scattered field $\vec{k}_{d i f f}$ :

$$
\vec{k}=\vec{k}_{d i f f}=\vec{k}_{i}+m \vec{G}
$$

with $m$ being an integer and $\vec{G}$ is the reciprocal lattice vector of the grating. Using a near-field tip instead of random defects, in order to probe information carried by the evanescent field, also removes the uncertainty about the scattering potential. It relies on the known transfer function of the tip and on the intrinsic spatial coherence of the information extraction process.
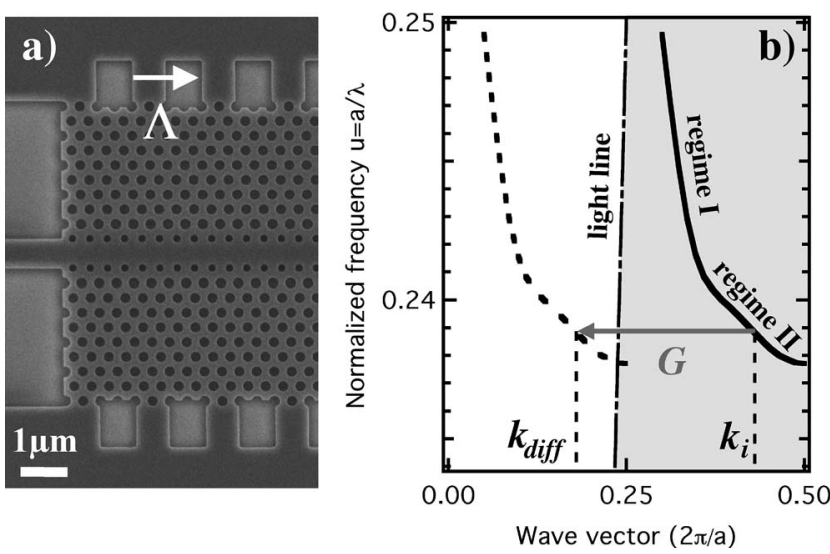

FIG. 1. (a) Electron microscopy image of a SW1 waveguide (see text) with the linear probe gratings at the waveguide boundaries. (b) Illustration of the folding of the theoretical SW1 dispersion curve (dark line) into the light cone (dash line). Gray region: region below the light cone.

We have combined the defect and near-field tip approaches by designing a linear probe grating (LPG) that probes the evanescent tail of a $\mathrm{PhC}$ Bloch mode. The photonic structures under investigation are conventional siliconon-insulator (SOI) W1 $\mathrm{PhC}$ waveguides ${ }^{16}$ and a special SOI W1 PhC waveguide (SW1) where the first two rows of holes adjacent to the $\mathrm{W} 1$ waveguide core have been modified to achieve a slow-light regime with a constant group index over a large frequency bandwidth, ${ }^{11}$ as shown in Fig. 1(a). The $\mathrm{Si}$ and $\mathrm{SiO}_{2}$ thicknesses are $338 \mathrm{~nm}$ and $1 \mu \mathrm{m}$, respectively. The diameter of the bulk holes is $234 \mathrm{~nm}$ and the diameters of the first and second rows of holes are decreased and increased by 54 and $9 \mathrm{~nm}$, respectively, compared to the bulk holes. The triangular PhC lattice has a pitch $a=370 \mathrm{~nm}$. As can be seen, a LPG was etched on both sides of the W1 guide at a separation of ten rows from the core. Each elementary cell of the LPG, consisting of a rectangular hole etched into the top silicon layer with a pitch $\Lambda=4 a$, acts as a local probe that scatters the transverse evanescent tails of the propagating mode.

Figure 1(b) illustrates the theoretical dispersion curve of the SW1 Bloch mode (dark line). The Bloch mode propagating in the SW1 lies below the light line indicated by the straight dash-dotted line. The slow-light regime with an extended bandwidth occurs at normalized frequencies $u$ between 0.238 and 0.241 with a group index $n_{g}$ around 30 (regime II), whereas for $u$ larger than 0.243 , the mode propagates with a group index around 5 (regime I). The scattering process, governed by the wave vector conservation rule [Eq. (2)] is illustrated with the arrow corresponding to $\vec{G}$. This representation shows that $G$ is large enough to have $\vec{k}_{\text {diff }}$ lying in the light cone. The selected pitch $\Lambda$ of the LPG is adapted to fold the first order $(m=1)$ of $S\left(\vec{k}, \vec{k}_{i}, \omega\right)$ into the light cone. As a result, the dispersion relations $\vec{k}_{i}(\omega)$ of the SW1 Bloch mode can be completely inferred from the knowledge of $\vec{k}_{\text {diff }}$ and $\vec{G}$. 


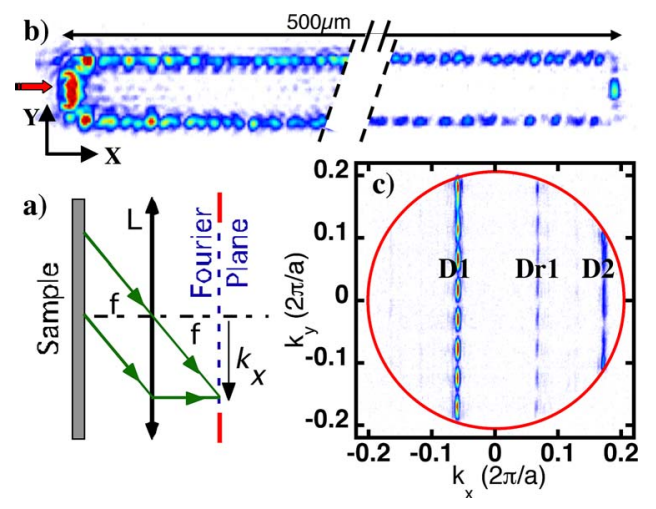

FIG. 2. (Color online) (a) Schematic of the Fourier imaging principle. (b) Real space near-infrared image of a $500 \mu \mathrm{m}$ SW1 waveguide $(\lambda=1514 \mathrm{~nm})$. (c) Corresponding far-field image. Red circle: Output pupil of the objective.

\section{EXPERIMENTAL RESULTS}

The emitted light is collected in the far-field from the sample surface with a high-numerical-aperture objective (NA $=0.9$ ) and imaged on a near-infrared charge coupled device camera. The excitation of the photonic structure is produced by a laser source tunable between 1.47 and $1.66 \mu \mathrm{m}$ with a narrow spectral linewidth of $0.001 \mathrm{~nm}$. The detection of the scattered light by the linear gratings, which act as a vanishingly weak perturbation, is made possible, thanks to the high sensitivity of the imaging setup. By design, the coupling of the LPG with the Bloch mode, and consequently, the resulting distortion of the mode dispersion, can be as low as desired.

The real space image of a $500 \mu \mathrm{m}$ long SW1 excited at $\lambda=1.514 \mu \mathrm{m}$ is shown in Fig. 2(b). An interference pattern with complex spatial modulations is observed at the linear grating position along the waveguide. At the line-defect position of the SW1, where the propagating Bloch mode is confined, no noticeable light is detected in the image as the propagation wave vector lies outside the light cone. The corresponding Fourier-space pattern in Fig. 2(c) is composed of sharp vertical lines, labeled D1, Dr1, and D2, that are perpendicular to the energy flow direction. As illustrated in Fig. 2(a), the Fourier image is located in the back focal plane of the collecting lens. Thanks to the conservation of the parallel component $k^{\|}$of the wave vector at the sample interface, there is a unequivocal correspondence between a point located at $\left(k_{x}, k_{y}\right)$ in this plane and the phase velocity of the wave radiated from the sample. The imaging plane size is restricted by the aperture of the collecting objective (red circle), associated with a collection angle of $130^{\circ}$, i.e., $k_{\max }^{\|}$ $=0.22(2 \pi / a)$. The diffracted lines observed in Fig. 2(c) originate from the out-of-plane scattering of the propagating W1 Bloch mode at the LPGs. They are perfectly straight $\left(k_{x}=\right.$ constant $)$ due to the one-dimensional (1D) symmetry of the gratings; this also illustrates the negligible geometrical aberrations of the optical system. When the angular frequency $\omega$ corresponds to the band gap region of the PhC W1 waveguide, they disappear, confirming that they are not related to leaky lateral surface modes or intrinsic eigenmodes
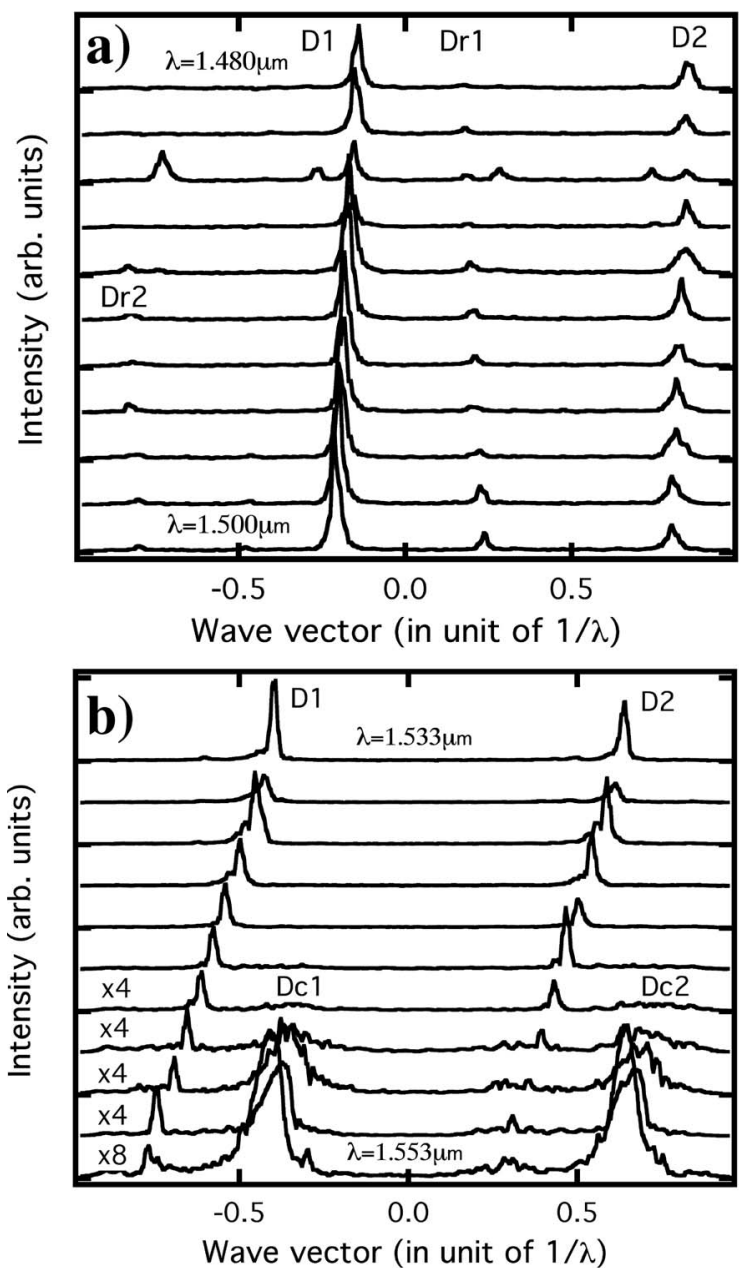

FIG. 3. [(a) and (b)] Far-field spectra of the SW1 waveguide at different wavelengths in regimes I and II, respectively. Each spectrum is spaced by $2 \mathrm{~nm}$ wavelength step.

of the LPGs, which would be directly excited from the interface at the W1 input. The periodic modulation observed along the vertical direction of the lines results from the farfield interference between the two linear gratings on each side of the waveguide. It is assessed by the absence of the modulation when the intensity emitted from one of the LPG is blocked in an intermediate real space imaging plane.

Figures 3(a) and 3(b) show the far-field angular spectra (intensity profiles of the Fourier-space images along the $k_{x}$ direction) of the SW1, corresponding to the two different regimes of the SW1 dispersion curve: regime I between 1.480 and $1.500 \mu \mathrm{m}$ (normal regime) and regime II between 1.533 and $1.553 \mu \mathrm{m}$ (slow-light regime). In regime I [Fig. 3(a)], the observed spectral features exhibit a slight positive spatial frequency shift with decreasing $u$ in the case of D1 and D2 and a negative one for Dr1 and Dr2. The spatial frequency gaps between the spectral lines D1 and D2, between Dr1 and Dr2, and between Dc1 and Dc2 [Fig. 3(b)] are exactly equal to the reciprocal lattice vector of the linear grating $G=2 \pi / \Lambda$. In regime II [Fig. 3(b)], the shift of the peaks D1 and D2 with decreasing $u$ drastically accelerates, Dr1 and Dr2 are absent, and an additional broad feature appears for an excitation wavelength above $1.544 \mu \mathrm{m}$. The D1 


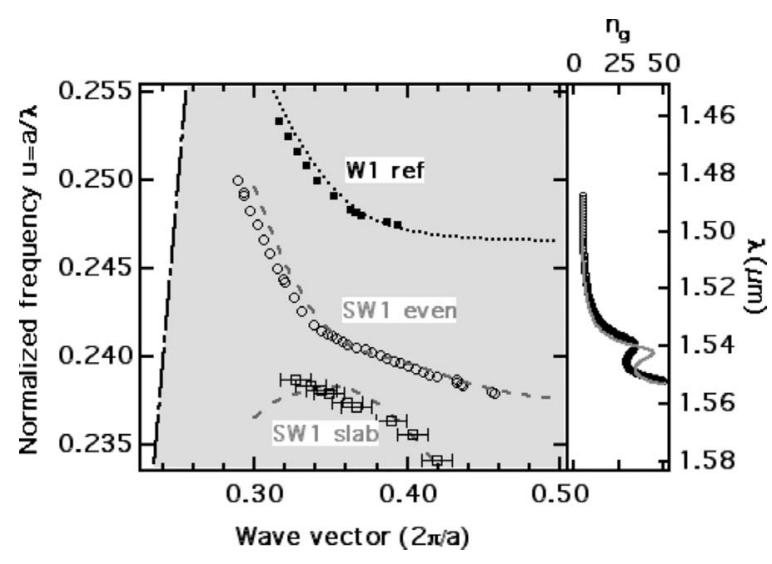

FIG. 4. Left: Experimental dispersion curves of the reference W1 waveguide mode (dark squares), of the SW1 even mode (open circles), and of the dielectric band (open squares), with error bars corresponding to the linewidth of the modal spectrum. The gray dashed curves and the dark dotted curve are theoretical 3D planewave expansion calculation of the SW1 and of the reference W1, respectively. Right: Theoretical (gray) and experimental (black) group-index dispersion curves.

and D2 peaks can be identified as the first $(m=1)$ and second $(m=2)$ diffracted orders of the W1 guided Bloch mode diffracted by the side coupled LPGs. The Dr1 and Dr2 lines are located at opposite $k$ position of D1 and D2, respectively, and are not separated from these last lines by the reciprocal lattice vector of the LPG. Moreover, the D1 and Dr1, as well as the D2 and Dr2, undergo an opposite $k$ variation with varying $u$, which is impossible for frequency components belonging to the same Bloch wave, as all the Fourier components of a Bloch mode have the same group velocity. It turns out that the sample has mirrorlike cleaved facets at each end (the total sample length is around $1.5 \mathrm{~mm}$ ). As a result of the reflectivity of these facets, Fabry-Pérot (FP) fringes appear in the transmission spectra through the complete structure ( $\mathrm{PhC}$ waveguide+access waveguides) that can be measured with conventional end fire setup. The Dr1 and Dr2 lines originate from the backward propagating Bloch mode resulting from the reflection at the end facet of the sample. Note that in the current $\mathrm{PhC}$ structure, the group velocity is positive for a wave scattered in free space with a negative wave vector. ${ }^{17,18}$ As a result, the first order D1 of the forward propagating wave D1 in the Fourier space is located at $-k_{x}$, i.e., its phase velocity is negative. The broad peaks Dc1 and Dc2 originate from dielectric band modes, i.e., from the emission of propagating Bloch modes excited in the $\mathrm{PhC}$ continuum. The wave vector spectrum at $\lambda$ $=1.484 \mu \mathrm{m}$ in Fig. 3(a) exhibits additional peaks near each spectral feature. These peaks come from the resonant excitation of a 1D Bloch wave of the LPG. In Fig. 3(b), the gradual decrease of the collected intensity of the D1 line in regime II comes mainly from the drop of the modal coupling efficiency at the SW1 input.

We have plotted in Fig. 4 the experimental dispersion curves of the SW1 and the reference W1 standard waveguide deduced from Fig. 3. The curve labeled "SW1 even" deduced either from the D1 peak or from the D2 peak and the curve labeled "SW1 slab" from the Dc1 or Dc2 peak. The theoretical dispersion relations calculated with the 3D planewave expansion method are in excellent agreement with the experimental dispersion relations. From this figure, we see that the entire first Brillouin zone of the $\mathrm{PhC}$ waveguide is explored, thanks to the use of the LPG probe. To infer the dispersion curve below the light line from the wave vector conservation rule [Eq. (2)], only the LPG parameter $\vec{G}$ is required. The figure on the right shows the experimental group-index dispersions curve $n_{g}(\omega)$, deduced from Fig. 4, which is in good agreement with the theoretical one.

In Fig. 4, the dispersion curves of the reference W1 waveguide mode and of the SW1 even mode are only plotted for wave vectors below $0.40(2 \pi / a)$ and $0.46(2 \pi / a)$. Beyond such limit values, which correspond to group index as high as $n_{g} \sim 100$, several extra lines appear in the far-field spectra. As a result, the dispersion curve of a true guided mode cannot be determined unambiguously. In addition, the field intensity profile along the LPGs, which is continuous for $n_{g}<100$, as shown in Fig. 2(b), becomes strongly discontinuous for $n_{g}>100$. We attribute such observations to the $\mathrm{PhC}$ structural disorder which induces localization in onedimensional structures. The limit of the experimental dispersion curves in Fig. 4 highlights the transition between a true propagating mode whose field pattern should extend along the whole $\mathrm{PhC}$ waveguide and a set of localized modes in the $\mathrm{PhC}$ waveguide due to disorder. The effect of nanometer scale disorder in one-dimensional slow-light waveguides has recently been investigated theoretically in Refs. 19 and 20. Such calculations lead to the conclusion that $100 \mathrm{~nm}$ wide waveguide requires $1 \mathrm{~nm}$ uniformity to achieve $n_{g}=100$ due to localization in agreement with our experimental results. We would like to point that the experimental determination of the group index $n_{g}$ in the very slow-light regime $\left(n_{g}>100\right)$, which is one of the main interesting parts of the dispersion curve, can lead to misleading interpretations due to the one-dimensional localization effect. As an example, the ATR technique proposed by Galli et al. ${ }^{14}$ is unable to distinguish between a true propagating slow mode and a localized mode due to the disorder. Probing a localized mode with ATR measurement would also result in a flatband, as observed in the paper of Galli et al. in the top right of Fig. 7. We believe that the group index as large as 1000 and the experimental determination of the dispersion curve up to Brillouin zone boundary $0.50(2 \pi / a)$ reported in Ref. 14 cannot be unambiguously attributed to a true propagating mode. In contrast, the imaging far-field technique that we propose is able to assess the onset of the localization-delocalization transition in photonic waveguides. Note also that up to now, the best slow-light group index achieved in W1 silicon waveguide, with state of the art technology by Vlasov et al., ${ }^{6}$ was as low as 150 in real condition of operation.

\section{CONCLUSION}

In conclusion, the design of a linear grating, acting as a near-field probe, enables us to recover with far-field optics the dispersion relation of Bloch waves propagating below the light cone. The fine spectral analysis provides a deep physi- 
cal understanding of the dispersion relation from experimental measure. Such a technique will be of great interest, e.g., in characterizing the effect of special designs of the dispersion curves on slow-light propagation or nonlinear interactions. This also demonstrates that the information carried by evanescent waves can be transferred in an efficient, robust, and accurate way into propagating waves, which is a key element in achieving an optical resolution beyond the classical diffraction limit. Finally, note that our approach demonstrated in the 1D case can be easily extended to two dimensions by superimposing a shallow etched probe grating directly on top of the object.

\section{ACKNOWLEDGMENTS}

This project was supported by the European Network of Excellence ePIXnet IST-004525, Funfox IST-004582, the Swiss Quantum Photonics National Center for Competence in Research (NCCR-QP), the Danish Technical Research Council through the research program PIPE, and the COST action P11. The authors would like to thank R. Rochat for his creative technical assistance, W. Langbein for encouraging discussion about $k$-space imaging, and L. A. Dunbar and B. Devaud-Plédran for careful reading of the paper.
${ }^{1}$ M. A. Foster, A. C. Turner, J. E. Sharping, B. S. Schmidt, M. Lipson, and A. L. Gaeta, Nature (London) 441, 960 (2006).

${ }^{2}$ S. I. Bozhevolnyi, V. S. Volkov, E. Devaux, J.-Y. Laluet, and T. W. Ebbesen, Nature (London) 440, 508 (2006).

${ }^{3}$ A. P. Hibbins, B. R. Evans, and J. R. Sambles, Science 308, 670 (2005).

${ }^{4}$ T. F. Krauss, R. M. De La Rue, and S. Brand, Nature (London) 383, 699 (1996).

${ }^{5}$ M. Notomi, K. Yamada, A. Shinya, J. Takahashi, C. Takahashi, and I. Yokohama, Phys. Rev. Lett. 87, 253902 (2001).

${ }^{6}$ Y. A. Vlasov, M. O'Boyle, H. F. Hamann, and S. J. NcNab, Nature (London) 438, 65 (2005).

${ }^{7}$ A. Yariv, Y. Xu, R. K. Lee, and A. Scherer, Opt. Lett. 24, 711 (1999).

${ }^{8}$ J. B. Khurgin, Opt. Lett. 30, 513 (2005).

${ }^{9}$ D. Mori and T. Baba, Opt. Express 13, 9398 (2005).

${ }^{10}$ A. Karalis, E. Lidorikis, M. Ibanescu, J. D. Joannopoulos, and M. Soljacic, Phys. Rev. Lett. 95, 063901 (2005).

${ }^{11}$ L. H. Frandsen, A. V. Lavrinenko, J. Fage-Pedersen, and P. I.
Borel, Opt. Express 14, 4357 (2006).

${ }^{12}$ M. L. M. Balistreri, H. Gersen, J. P. Korterik, L. Kuipers, and N. F. van Hulst, Science 294, 1080 (2001).

${ }^{13}$ H. Gersen, T. J. Karle, R. J. P. Engelen, W. Bogaerts, J. P. Korterik, N. F. van Hulst, T. F. Krauss, and L. Kuipers, Phys. Rev. Lett. 94, 123901 (2005).

${ }^{14}$ M. Galli, D. Bajoni, M. Patrini, G. Guizzetti, D. Gerace, L. C. Andreani, M. Belotti, and Y. Chen, Phys. Rev. B 72, 125322 (2005).

${ }^{15}$ A. Sentenac, P. C. Chaumet, and K. Belkebir, Phys. Rev. Lett. 97, 243901 (2006).

${ }^{16}$ S. J. McNab, N. Moll, and Y. A. Vlasov, Opt. Express 11, 2927 (2003).

${ }^{17}$ P. St. J. Russell, Appl. Phys. B: Photophys. Laser Chem. 39, 231 (1986).

${ }^{18}$ B. Lombardet, L. A. Dunbar, R. Ferrini, and R. Houdr, J. Opt. Soc. Am. B 22, 1179 (2005).

${ }^{19}$ S. Mookherjea, J. Opt. Soc. Am. B 23, 1137 (2006).

${ }^{20}$ S. Mookherjea, A. Oh, Opt. Lett. 32, 289 (2007). 